\title{
ICC XV, 15th International Chromosome Conference
}

\author{
5-10th September, 2004, Brunel University
}

This issue of Cytogenetic and Genome Research is published on the occasion of ICC XV, the 15th International Chromosome Conference held at Brunel University on 5-10 September 2004. The majority of the enclosed papers represent the nominees for the prizes in several categories at the conference. All nominees, as part of the selection process, were required to submit a manuscript to this special issue and, while the enclosed work may not cover the entirety of their presentation, it will give you, at least, a flavour of on what they are working. A broad range of cytogenetic research and different organisms are covered here. Looking over the papers as a whole, the authors, collectively, seem to have leaned toward technological developments. The beautiful multicolour meiotic preparations of Codina-Pascual and colleagues sit well alongside the Atomic force microscopy of Takeyasu et al. (in prokaryotes) and Hoshi et al. in human chromosomes. Silahtaroroglu et al. demonstrate the use of Locked Nucleic Acid (LNA) probes in place of DNA or PNA probes for FISH experimentation while Uchiyama et al. use 2D electrophoresis to analyse the protein composition of human chromosomes. Laner et al. follow our Brunellian theme demonstrating the use of a human artificial chromosome generated from PAC constructs containing artificial centromeres and telomeres and all this work is supplemented by Bailey et al. who present a very nice review of the applications of $\mathrm{CO}$ FISH.

Scientific questions are not left behind however with Lightfoot and Hoog presenting a study of embryos from a model "Scyp3-null" mouse that produces aneuploid oocytes and subsequent embryos after fertilisation with normal sperm. Rotkov et al. give us an analysis of telomeres in Asparagales while Herron et al., in studying grasshopper B chromosomes, propose that they persist in evolution via a metaphase checkpoint alteration that, in turn was more forgiving of misaligned chromosomes. The evolutionary theme is continued by Ferreri et al. with an evolutionary study of the centromere in marsupials drawing general conclusions about the role of this vital structure in chromosome evolution. We look forward to hearing all of their presentations at ICC XV and to learning who will be the prize winners chosen by the International Advisory Panel.
In this issue there are also several specially selected articles that complement the ICC XV contributions. An animal chromosome/comparative genomics theme predominates with several authors assigning and characterizing genes and non-coding sequences in various species. There is one epigenetic contribution (imprinting regions in mouse) and three in the human disease/cancer area i.e. supernumerary chromosomes, inversion breakpoint localization and microarray $\mathrm{CGH}$.

The International Chromosome conferences began life in 1964 as the Oxford Chromosome Conferences and adopted the "International" tag at the fourth conference in Jerusalem in 1972. The tri-annual format has continued since the inception and the international flavour has been maintained with conferences going on in Israel (once), the Netherlands (once), Scandinavia (twice), France (once), Italy (once), Spain (once), Germany (twice) and the UK a total of five times (including the initial 3 Oxford Chromosome Conferences and the valedictory return in 1980). The year 2004 sees another return to the UK, this time to Brunel University. The decision to hold ICC XV at Brunel was initiated in a Franconian wine cellar at The Residence in Würzburg at the 14th International Chromosome Conference in 2001. Competition was significant however we'd like to think that Brunel was chosen because of its conference facilities and proximity to Heathrow Airport. Delegates will benefit from being only 15-20 minutes away from their arrival and departure gates and we hope to house the majority of delegates on campus for the duration of the conference. Brunel University is a large self-contained campus to the west of London. It is known locally for its landscaped gardens, "brutalist" architecture and excellent hospitality. It has over 12,000 students and over 850 academic staff and was named after the celebrated Victorian engineer, Isambard Kingdom Brunel. UK readers will remember IKB leading the "greatest Briton" poll for the majority of the long-running saga before being usurped by Winston Churchill at the eleventh hour.

At ICC XV we wish to create an overall feel of not only top quality science but the ability for delegates to discuss new ideas and projects in a relaxed setting. It is often said that the best science is not done in the lecture rooms but in the bars and 
social areas of conference centres. The International Chromosome Conferences have always promoted the social side of conferencing as well as the scientific side and ICC XV will be no exception. We will be organising visits to the West End, Windsor Castle, Virginia Water, the Tate Modern Gallery and the London Eye. Events on-campus will include real ale tasting, a FISH and chip supper, film shows and a musical recital and for the conference dinner - a medieval banquet, with traditional dancing. We were delighted that, following our initial requests, our "wish-list" of speakers was fulfilled. One of the previous criticisms of the International Chromosome Conferences was that it was heavily biased towards European research. We would like to think that we have addressed this by attracting large contingents from the US, Asia and Australasia. There are around 120 invited speakers and approximately 100 of submitted abstracts have been selected as oral presentations. In addition there will be two poster sessions, collectively comprising around 150 posters accompanying our exhibition space. With luck, we hope to have over 700 delegates, each contributing to the unique nature of ICC. We would like to express our appreciation for the efforts of senior management at Brunel for listening to our complaints when the campus building work threatened to impede our progress, to Brunel Conferencing Services for arranging the room bookings and catering and, especially to Cambridge Publications for their organisational and financial management skills.

Cytogenetic research is lucky to have had a number of eras that have laid down fundamentals for several generations on biological and biomedical enquiry. For this reason our first plenary session will celebrate this. It is our opinion that three eras of cytogenetic research have graced the scientific literature over the years; the first being the classical era. The discovery of the correct number of human chromosomes and the correlations between numerical aberrations and disease (such as Down Syndrome and Klinefelter Syndrome) brought cytogenetic research into the forefront of science in the late 1950s and 1960s. As our plenary speaker to represent the classical era, we approached Professor Pat Jacobs who was involved in the discovery of the genetic basis of what we now know as sex chromosome abnormalities and her activities continue to this day. The ability to band human chromosomes brought chromosome research into a new era, enabling the detection of subtle rearrangements and heralding the advent of prenatal diagnosis and cancer cytogenetics. The name that is most synonymous with chromosome banding and our plenary speaker for the banding era is Dr. Adrian Sumner who is also the chair of the standing committee for ICC. Finally our decision about whom to represent the renaissance of cytogenetics, namely the FISH era, was clearly Professor David Ward. In a time when molecular biologists were largely writing the obituaries for cytogenetics, the technique of FISH was developed. At the forefront of this revolution was Professor Ward's laboratory.

We would like to propose that we are now entering our fourth era, namely that of chromosome engineering. The Brunellian overtones of this suggestion are not lost on us but we would argue that they are well founded given the extensive interest in gene therapy, gene function, genome organisation and chromosome transfer, all of which derive their origins from cytogenetic research. To this end our second plenary session will be based around chromosome engineering. We are delighted to have attracted some of the leading lights in the field of chromosome engineering, all of whom lead the world in the development and utility of human and mammalian artificial chromosomes. They are Professor Andy Choo of Melbourne, Australia, Dr Christine Farr of the University of Cambridge, UK and Professor Huntington F. Willard of Duke University, USA. All have agreed to speak at the I.K. Brunel plenary session on chromosome engineering. There will also be many other delegates from similar research areas involved in various sessions at the ICC XV. Our plenary speakers have been instrumental in moving the chromosome engineering field forward to a place where we can see similar vectors created by these researchers used in 21 st century therapies. The artificial chromosomes constructed by these eminent speakers are either created by the "top down" method developed in the laboratory of Dr Farr. This method uses telomere-associated chromosome fragmentation to produce minichromosomes from larger normal chromosomes. Conversely, the so-called "bottom up" method requires the minimal DNA sequences needed to make a chromosome to be placed into nuclei, where some cells will produce functional artificial chromosomes. Professor Willard's laboratory was the first to demonstrate the power of this technique. Professor Choo was the first to prove the existence of neocentromeres, regions of chromosomes that form functional centromeres without alpha satellite DNA and use them to engineer novel human artificial chromosomes. We look forward to hearing the latest advances from these three plenary chromosome engineers and to all the other advances made by other chromosome engineers in all the other sessions.

Our keynote speaker will be Professor John Burn of the University of Newcastle. Professor Burn is the University Orator at Newcastle as is well known for his television appearances and futuristic vision. We look forward with great enthusiasm to his aptly entitled presentations "The Future of Genetics." The remainder of our main sessions will be based around aneuploi$\mathrm{dy}$, chromosome evolution, genome organisation, sex chromosomes, tumour cytogenetics, meiosis, centromeres, telomeres, epigenetics, reproduction, prokaryote chromosomes, plant chromosomes, animal chromosomes, gene therapy, the postgenomic era and new methodologies. Sessions will be a mixture of invited speakers and selected abstracts and there will be plenty of time allocated for the viewing of posters and the exhibition as well as the obligatory socialising. So, in conclusion, at Brunel we are gathering some of the world's best scientists to celebrate the successes of cytogenetic research and look to its future achievements. We plan an outstanding scientific and social programme and even a session on chromosome engineering, we'd like to think that Isambard K. Brunel would have been proud.

\author{
Darren K. Griffin and Joanna M. Bridger \\ Cell and Chromosome Biology Group \\ Department of Biological Sciences, Brunel University \\ West London UB8 3PH (UK)
}

